\title{
COMPARAÇÃO DAS AVALIAÇÕES NEUROPSICOLÓGICAS EM MENINA COM DOENÇA CEREBROVASCULAR BILATERAL (MOYAMOYA) ANTES E APÓS A INTERVENÇÃO CIRÚRGICA
}

\author{
SYLVIA MARIA CIASCA*, HÉLVIO LEITE ALVES**, \\ INESS ELCIONE GUIMARÄES ${ }^{* * *}$, ANA PAULA CASTRO TERRA ${ }^{* * *}$, \\ M. VALERIANA L. MOURA-RIBEIRO****, EDWALDO E. CAMARGO***** \\ ELBA SÁ CAMARGO ETCHEBEHERE******, ALLAN DE OLIVEIRA SANTOS******
}

\begin{abstract}
RESUMO - A doença de moyamoya é anormalidade cerebrovascular crônica e progressiva identificada através das características angiográficas; estão presentes no quadro clínico episódios isquêmicos transitórios, cefaléia, crises convulsivas, hemiparesia, que podem desaparecer após tratamento cirúrgico. Nós descrevemos o caso de uma menina com características clássicas da doença, comparando-o em dois momentos, antes e depois da cirurgia, através de avaliações neurológicas, neuropsicológicas, e exames complementares.
\end{abstract}

PALAVRAS-CHAVES: moyamoya, doença cerebrovascular, criança, avaliação neuropsicológica, SPECT.

Comparison of the neuropsychological assessment in a girl with bilateral cerebrovascular disease (moyamoya) before and after surgical intervention

ABSTRACT - Moyamoya is a chronic progressive cerebrovascular disease with characteristic angiographic findings and a clinical picture with episodes of transient ischemic attacks, headache, seizures, hemiparesis, which may resolve after surgical treatment. We describe the case of a girl with the typical findings of the disease, comparing them before and after surgery with the use of neuropsychological tests, neurological examination and laboratory tests.

KEY WORDS: moyamoya, cerebrovascular disease, child, neuropsychological assessment, SPECT.

A doença cerebrovascular moyamoya é descrita como doença crônica e progressiva com características angiográficas típicas. O termo moyamoya tem origem japonesa e significa "algo nebuloso, como cortina de fumaça". Em sua maior parte, os casos descritos na literatura são do Japão. Entretanto, a doença tem distribuição universal. Metade dos casos descritos é de crianças, muitas delas na idade pré-escolar ${ }^{1}$. Nesta doença ocorre oclusão bilateral das artérias carótidas internas e, pelo fato de a oclusão ser lenta e progressiva, surgem anastomoses múltiplas que se formam entre as artérias carótidas internas e externas, compostas pelas colaterais das artérias coroidais anterior e posterior, artéria basilar, além de artérias meníngeas ${ }^{2,3}$. A etiologia é desconhecida e a maioria dos casos não apresenta fator predisponente. Porém, a hereditariedade é defendida , pois há estudos que mostram incidência familiar em $7 \%$ dos $\operatorname{casos}^{4}$. O quadro clínico é caracterizado por episódios isquêmicos transitórios, cefaléia, crises convulsivas, hemiparesia que pode ter involução parcial ou total. A criança fica letárgica, comatosa, sendo algumas vezes detectadas hemianopsia, hemianestesia e afasia. Algumas crianças têm coréia de face e de membros ${ }^{1}$.

Hospital das Clínicas (HC) da Faculdade de Ciências Médicas (FCM) da Universidade Estadual de Campinas (UNICAMP): *Professor Assistente; **Neurocirurgião; ***Aprimoranda; ****Professor Adjunto, Disciplina de Neurologia Infantil; *****Professor Dr. Diretor do Serviço de Medicina Nuclear; ******Assistente, Serviço Medicina Nuclear. Aceite: 5-agosto-1999.

Dra. Sylvia Maria Ciasca - Praça XV de Novembro 40/41 - 13024-180 Campinas SP - Brasil. 
$\mathrm{Na}$ doença de moyamoya, o estudo angiográfico fornece o diagnóstico, envolvendo estenose de vasos do circuito arterioso e colaterais tálamo-estriados, com aspecto característico de estrias esfumaçadas ${ }^{1,4,5}$. Zonas de infarto parenquimentoso podem ser observadas nos exames de tomografia computadorizada e ressonância magnética. O SPECT cerebral mostra áreas de hipoperfusão, e no EEG observa-se o aparecimento de ondas de alta voltagem um minuto após o término da hiperpnéia, além de alentecimento hemisférico ou generalizado da atividade elétrica cerebral. O Doppler transcraniano é procedimento útil, com a constatação dinâmica das modificações do fluxo sanguíneo regional ${ }^{4,6,7}$. O tratamento é cirúrgico, dirigido no sentido de promover novas anastomoses entre os territórios carotídeos extra e intra-cranianos e apresenta resultados positivos em mais de $50 \%$ dos casos. O prognóstico é pobre e a maioria dos casos continua a apresentar fraqueza crônica de um ou de ambos os lados, epilepsia e retardo mental em graus variados.

Neste estudo, procurou-se correlacionar os dados obtidos através das avaliações neurológica, funcional e neuropsicológica de um quadro clínico da doença cerebrovascular bilateral (moyamoya), em dois momentos: pré-cirúrgico ( $1^{\mathrm{a}}$ avaliação) e pós-cirúrgico ( $2^{\mathrm{a}}$ avaliação). A realização das avaliações proporcionou uma análise qualitativa da produção da criança conseguindo elementos para pesquisa de praxias, gnosias, linguagem, memória e processos intelectuais ${ }^{8}$.

\section{MÉTODO}

Criança do sexo feminino, nível sócio-econômico médio, com 9 anos e 7 meses na $1^{\text {a }}$ avaliação (précirúrgica), frequentando a $2^{\mathrm{a}}$ série do $1^{\mathrm{o}}$ grau, em Escola Pública da região de Campinas, apesar de não ser alfabetizada. $\mathrm{Na} 2^{\mathrm{a}}$ avaliação (pós-cirúrgica) a criança tinha 10 anos e 4 meses, e havia sido transferida para classe especial em outra Escola Pública da mesma região, continuando sem alfabetização completa.

Nas duas avaliações realizadas utilizaram-se: a) Escala Wechsler de Inteligência para Criança (WISC), com o objetivo de medir quantitativamente e qualitativamente o nível cognitivo geral; b) Teste Guestáltico VisoMotor (Bender), para avaliação do grau de amadurecimento perceptivo viso-motor; c) Bateira Luria Nebraska para Criança (BLN), com o objetivo de avaliar dez áreas neuropsicológicas relacionadas com áreas corticais específicas; d) Exame Neurológico Tradicional (ENT); e) Angiografia Carotídea; f) Single Photon Emission Computed Tomography (SPECT), com imagens cerebrais obtidas após injeção venosa de 20mCi de HMPAO99mTc para avaliar fluxo sanguíneo cerebral.

A coleta de dados foi realizada em três fases:

1) Realizou-se a primeira avaliação no Ambulatório de Distúrbio de Aprendizagem da FCM/UNICAMP, com cinco consultas, duração média de 50 minutos, para aplicação dos instrumentos avaliativos antes da cirurgia.

2) Técnica cirúrgica: auto-transplante de omento vascularizado para região intracraniana. A paciente foi submetida a incisão coronariana fronto-temporal esquerda com preservação da artéria temporal superficial em toda a sua extensão, seguida de craniotomia fronto-temporal. Procedeu-se à dissecção microcirúrgica desta artéria numa extensão de $20 \mathrm{~mm}$. Concomitantemente, o cirurgião pediátrico realizou minilaparotomia, retirando porção ampla do omento maior junto à grande curvatura do estômago, contendo a artéria gastroepiplóica. Realizou-se, então, arteriotomia na parede lateral da artéria temporal superficial anastomosando-se esta artéria à extremidade da artéria gastroepiplóica (anastomose término-lateral) usando-se fios de nylon 10-0, com microscópio cirúrgico. Observandose a patência da anastomose diretamente e através da extremidade sangrante do omento, procedeu-se à eletrocoagulação destes vasos em suas porções distais. Após dissecção microcirúrgica ampla da aracnóide que reveste a cisterna silviana e o córtex fronto-temporal adjacente, acomodou-se o omento vascularizado por sobre esta área. A seguir procedeu-se ao fechamento da dura-mater, recolocação do retalho ósseo, e fechamento da pele?

3) A criança foi submetida à segunda avaliação neuropsicológica com os mesmos instrumentos, utilizados na primeira avaliação, seis meses após o procedimento cirúrgico, no Ambulatório de Distúrbio de Aprendizagem da FCM/UNICAMP.

\section{RESULTADOS}

A criança é a primeira filha de um casal jovem, não consanguíneo. Gestação e nascimento sem intercorrências. Desenvolvimento neuropsicomotor pregresso adequado para a idade e sono tranquilo. Quando iniciou na pré-escola, a professora percebeu que a criança apresentava uma pressão muito forte na coordenação motora fina. Aos 7 anos começou apresentar dificuldades de coordenação motora e alteração da marcha; seguindo, teve alteração na linguagem, crises convulsivas, mudança 


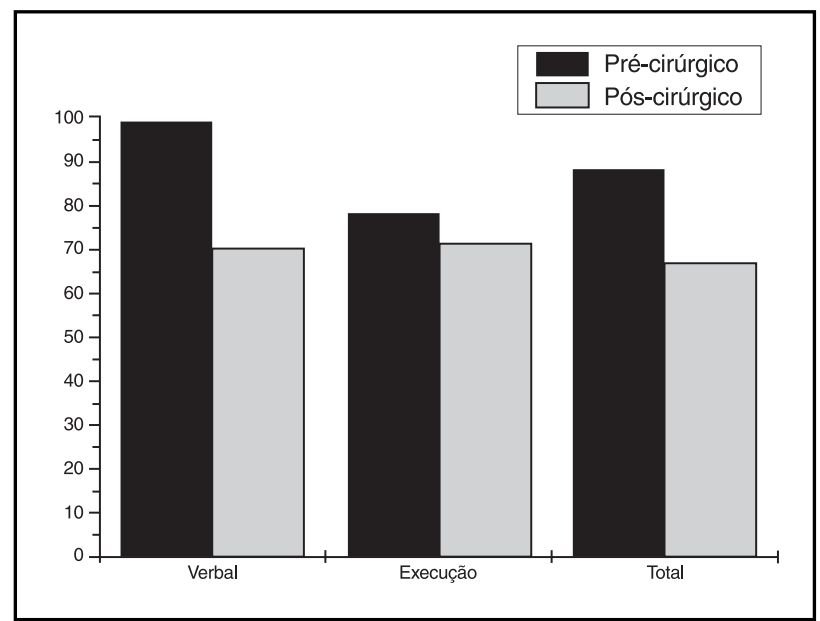

Fig 1. Doença de moyamoya. valores brutos obtidos nas duas avaliações no Teste WISC.

Tabela 1. Doença de moyamoya: avaliação cognitiva pelo WISC.

\begin{tabular}{lcc}
\hline & $1^{\mathrm{a}}$ avaliação & $2^{\mathrm{a}}$ avaliação \\
\hline Verbal & 99 & 70 \\
Execução & 78 & 71 \\
Total & 88 & 67 \\
\hline
\end{tabular}

de comportamento, humor e labilidade emocional.

Os dados obtidos referentes as condições pré-cirúrgica ( $1^{\mathrm{a}}$ avaliação) e pós-cirúrgica ( $2^{\mathrm{a}}$ avaliação) demonstraram:

Rendimento Intelectual - Significativa perda cognitiva entre uma avaliação e outra, tendo a Escala Verbal maior comprometimento do que a Escala Execução (Tabela 1 e Fig 1).

Bateria Luria Nebraska - Observou-se melhora significativa pós-cirúrgica nas áreas

Tabela 2. Doença de moyamoya: resultados da Bateria Luria Nebraska.

\begin{tabular}{|c|c|c|c|}
\hline & $1^{\text {a }}$ Avaliação & 2a Avaliação & Localização \\
\hline 1. Habilidades motoras & $75 \%$ & $100 \%$ & Córtex motor. Frontal parietal e temporal \\
\hline 2. Ritmo & $80 \%$ & $33 \%$ & Temporal \\
\hline 3. Habilidade tátil & $75 \%$ & $75 \%$ & Córtex sensitivo e regiões parieto occipital \\
\hline $\begin{array}{l}\text { 4. Tarefas perceptivas } \\
\text { (Bender) }\end{array}$ & $\begin{array}{l}\text { Abaixo } \\
\text { da média }\end{array}$ & $\begin{array}{l}\text { Abaixo } \\
\text { da média }\end{array}$ & $\begin{array}{l}\text { Occipital posterior HD e região parietal } \\
\text { anterior incomum no HE }\end{array}$ \\
\hline 5. Linguagem expressiva & $85 \%$ & $95 \%$ & Frontal temporal e parietal \\
\hline 6. Linguagem receptiva & $100 \%$ & $100 \%$ & Frontal secundário HE \\
\hline $\begin{array}{l}\text { 7. Memória imediata } \\
\text { a aprendizagem }\end{array}$ & $75 \%$ & $58 \%$ & Parietal HE \\
\hline 8. Raciocínio matemático & $83 \%$ & $50 \%$ & Região terciária parietal occipital temporal HE \\
\hline 9. Leitura & $30 \%$ & $30 \%$ & $\begin{array}{l}\text { Região terciária parietal occipital HE e ou HD } \\
\text { e lobo temporal }\end{array}$ \\
\hline 10. Escrita & $53 \%$ & $53 \%$ & $\begin{array}{l}\text { Região terciária lobo temporal parietal } \\
\text { occipital HE }\end{array}$ \\
\hline 11. Lateralidade & Destra & Sinistra & \\
\hline
\end{tabular}

HE, hemisfério esquerdo; HD, hemisfério direito. 


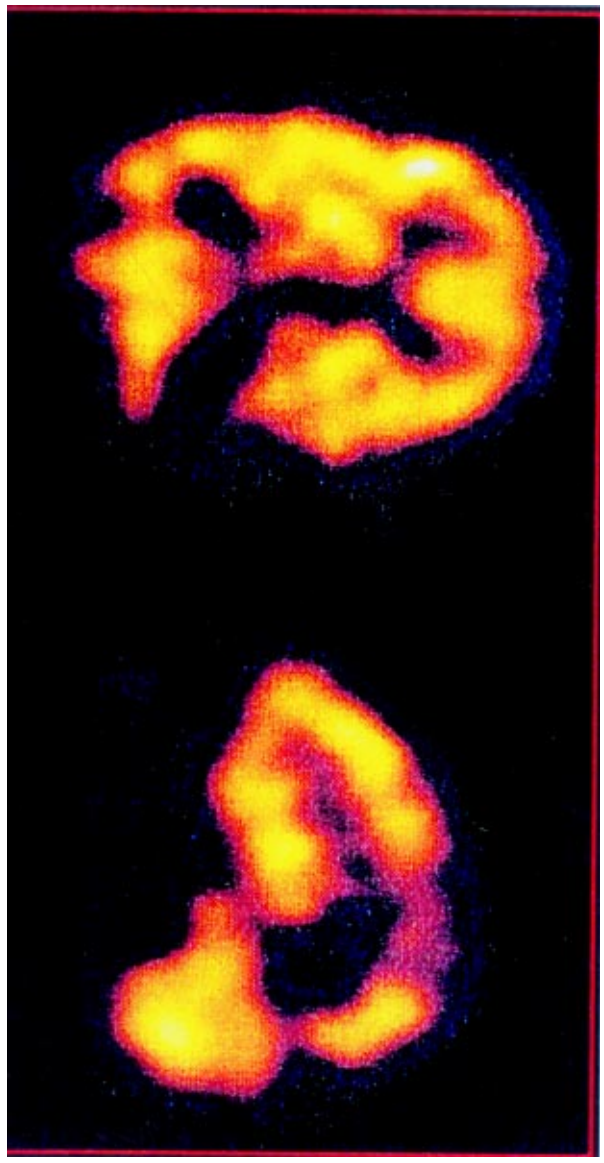

Fig 2. Doença de moyamoya. Corte transversal e sagital (pré-cirúrgico).

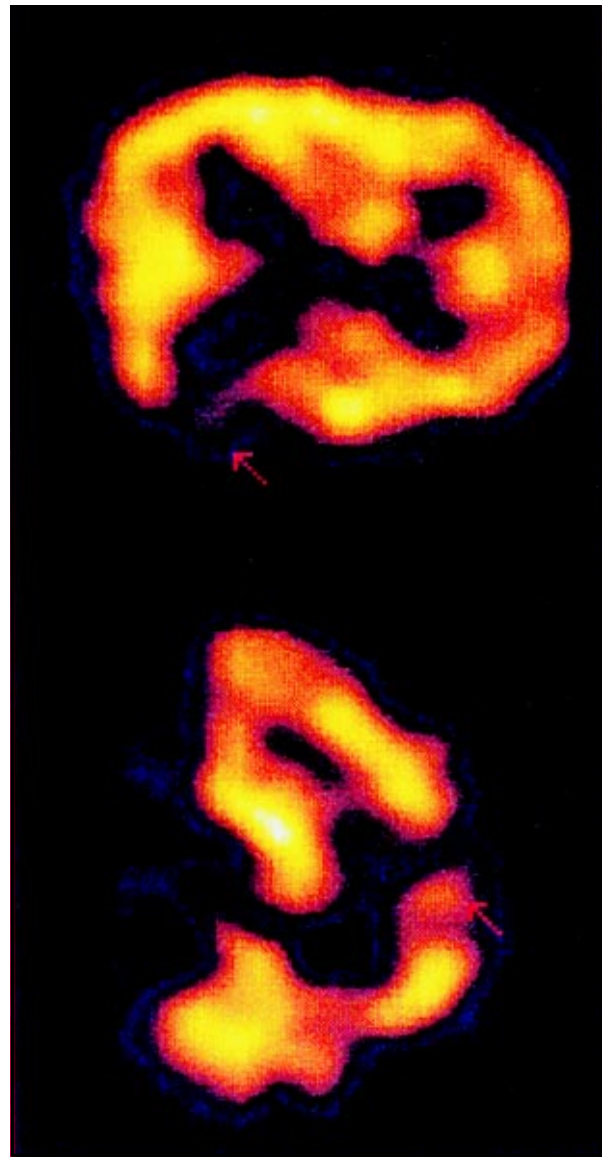

Fig 3. Doença de moyamoya: corte transversal $e$ sagital (pós-cirúrgico).

Tabela 3. Doença de moyamoya: resultados do SPECT pré e pós -cirúrgico.
$1^{\text {a }}$ avaliação
Hipoperfusão temporo-parietal E;
Área de hipoperfusão parieto-occiptal D.
$2^{\mathrm{a}}$ avaliação
Melhora da perfusão parietal E.

E,esquerdo; D, direito.

que envolvem habilidade motora no hemicorpo contralateral à hemiparesia e da linguagem expressiva. Houve perda de desempenho real, significativa nas provas de ritmo, em torno de $47 \%$, seguida de memória imediata para aprendizagem ( $17 \%$, em relação à primeira avaliação). Na percepção-motora, os escores foram abaixo dos esperados para sua faixa etária, caracterizando incapacidade de reprodução ou cópia de modelo visual. Aplicando-se provas que envolvem características básicas para aquisição das habilidades de leitura, escrita e raciocínio matemático compatíveis com o nível escolar da criança, notou-se perda de desempenho de raciocínio matemático em torno de $33 \%$, o mesmo não acontecendo nas habilidades necessárias para leitura e escrita. (Tabela 2).

Os resultados do SPECT (20-novembro-1997 e 20-agosto-1998) mostraram discreta melhora pós-cirúrgica (Tabela 3 e Figs 2 e 3 ). 


\section{DISCUSSÃO}

Em nosso estudo os resultados obtidos mostraram maior comprometimento no que se refere às habilidades cognitivas, quando correlacionados os dados obtidos através das avaliações neuropsicológicas anterior e posterior à cirurgia. Porém foi possível constatar melhora em provas que envolvem habilidade motora e linguagem expressiva, cujas áreas foram beneficiadas pela conduta cirúrgica de promoção de novas anastomoses do território carotídeo.

A atividade cortical da memória pode estar alterada quando ocorre uma mudança cerebral geral; observa-se portanto queda nos escores relacionados à memória imediata, possivelmente como resultado de tal atividade, confirmando que lesões na região parietal do hemisfério esquerdo podem produzir dificuldades na memória verbal auditiva de curta duração ${ }^{10}$.

Os baixos resultados nas provas raciocínio matemático podem estar relacionados ao fato de a criança não estar alfabetizada, uma vez que o quadro sintomático da doença teve início concomitantemente com o processo de alfabetização. Além disso, devido aos comprometimentos motores e de linguagem, a criança foi afastada do contexto escolar, retornando posteriormente a um nível de ensino inferior ao início da escolarização (classe especial).

As dificuldades verificadas na reprodução de estruturas e adaptação a ritmos implica em possível disfunção do lobo temporal, e a incapacidade de reproduzir ou copiar um modelo visual nas tarefas perceptivas, podem estar associadas a lesão ou disfunção parieto-occipital do hemisfério direito.

No primeiro SPECT cerebral, foi possível constatar, nos cortes transversal e sagital do exame pré-cirúrgico, extensa área de ausência de concentração de radiofármaco nos lobos temporal e parietal esquerdos (Fig 2), correlacionadas com as áreas de défict descritas. No segundo exame, verificou-se discreta melhora da perfusão no lobo parietal esquerdo (Fig 3) concordante com a melhora da função da linguagem expressiva, e habilidades motora conforme a bateria utilizada.

Torna-se claro através dos resultados obtidos que a melhora em determinadas funções não é necessariamente concomitante com o fator cognitivo geral, uma vez que inteligência requer não só adequação das áreas e funções, mas integração geral de capacidades diversas, exigindo interação de diversos fatores físicos e ambientais, entre outros.

A associação das avaliações neuropsicológica, neurológica e exames complementares por imagem possibilitou maior compreensão e clareza do diagnóstico, permitiu intervenção mais adequada para melhorar a aprendizagem geral e o prognóstico da paciente.

\section{REFERÊNCIAS}

1. Gherpelli JLD. Afecções vasculares cerebrais. In Diament A., Cypel S. Neurologia Infantil. 3Ed. São Paulo: Atheneu, 1996:1211-1212.

2. Fenichel GM. Neurologia pediátrica. 2Ed. Gadia C; Vissoky J, tradutores: Porto Alegre: Artes Médicas, 1995: 281-282.

3. Fodstad H, Bodosi M, Forssel A, Perricone D. Moyamoya disease in patients of Finno-Ugric origin. Br J Neurosurg 1996;10:179-186.

4. Ganesan V, Isaac E, Kirkham FJ. Variable presentation of cerebrovascular disease in monovular twins. Developl Med Child Neurol 1997;39:628-631.

5. Siqueira-Neto JI, Silva GS, Castro JDV, Santos AC. Neurofibromatose associada a arteriopatia de moyamoya e aneurisma fusiforme. Arq Neuropsiquiatr 1998;56:819-823.

6. Moura-Ribeiro MVL. Doença cerebrovascular em crianças e adolescentes. In Gagliardi RJ, Reimão R. Clínica neurológica. Sao Paulo. Lemos Editorial, 1998:97-102.

7. Muttagin Z. Cerebral circulation in moyamoya disease: a clínical study using transcranial Doppler sonography, Surg Neurol 1993;40:306-313.

8. Lefèvre BH. Neuropsicologia infantil. São Paulo: Sarvier, 1989.

9. Touho H, Karasawa J, Tenjin H, Ueda S. Omental transplantation using a superficial temporal artery previously used for encephaloduroarteriosynangiosis. Surg Neurol 1996;45:550-558.

10. Xavier GF. Memória: correlatos anátomo-funcionais. In Nitrini R, Caramelli P, Mansur L. Neuropsicologia: das bases anatomicas à reabilitação. São Paulo: Clínica Neurológica do Hospital das Clínicas da Faculdade de Medicina da USP, 1996. 